\title{
Taurine-upregulated gene 1: A vital long non-coding RNA associated with cancer in humans (Review)
}

\author{
WEN-YU WANG ${ }^{1 *}$, YAN-FEN WANG ${ }^{2 *}$, PEI MA ${ }^{1}$, TONG-PENG XU ${ }^{1}$ and YONG-QIAN SHU \\ ${ }^{1}$ Department of Oncology, The First Affiliated Hospital of Nanjing Medical University, Nanjing, \\ Jiangsu 210029; ${ }^{2}$ Department of Pathology, The Affiliated Hospital of Yangzhou \\ University, Yangzhou University, Yangzhou, Jiangsu 225000, P.R. China
}

Received July 27, 2016; Accepted July 6, 2017

DOI: $10.3892 / \mathrm{mmr} .2017 .7472$

\begin{abstract}
It is widely reported that long non-coding RNAs (lncRNAs) are involved in regulating cell differentiation, proliferation, apoptosis and other biological processes. Certain lncRNAs have been found to be crucial in various types of tumor. Taurine-upregulated gene 1 (TUG1) has been shown to be expressed in a tissue-specific pattern and exert oncogenic or tumor suppressive functions in different types of cancer in humans. According to previous studies, TUG1 is predominantly located in the nucleus and may regulate gene expression at the transcriptional level. It mediates chromosomal remodeling and coordinates with polycomb repressive complex 2 (PRC2) to regulate gene expression. Although the mechanisms of how TUG1 affects the tumor genesis process remain to be fully elucidated, increasing studies have suggested that TUG1 offers potential as a diagnostic and prognostic biomarker, and as a therapeutic target in certain types of tumor. This review aims to summarize current evidence concerning the characteristics, mechanisms and associations with cancer of TUG1.
\end{abstract}

\section{Contents}

1. Introduction

2. TUG1 in human cancer

3. Conclusions and perspectives

Correspondence to: Dr Yong-Qian Shu or Dr Tong-Peng Xu, Department of Oncology, The First Affiliated Hospital of Nanjing Medical University, 300 Guangzhou Road, Nanjing, Jiangsu 210029, P.R. China

E-mail: yongqian_shu@163.com

E-mail: tongpeng_xu_njmu@163.com

*Contributed equally

Key words: long noncoding RNAs, taurine-upregulated gene 1, cancer

\section{Introduction}

Long non-coding RNAs (lncRNAs) are characterized as a subgroup of RNAs $>200$ nucleotides in length without, or with limited, protein-coding potential (1-3). Previous studies have demonstrated that certain lncRNAs have regulatory roles in diverse biological processes at the epigenetic, transcriptional and post-transcriptional levels due to their various structural and biochemical characteristics (4-9). The aberrant expression of lncRNAs has been shown in various human diseases, including cancer (Table I).

Taurine-upregulated gene 1 (TUG1), a 7.1-kb lncRNA, was originally detected in a genomic screen for genes upregulated in response to taurine treatment in developing mouse retinal cells (10). It is located on chromosome 22 q12.2 in the human genome, and has been reported to be expressed in a tissue-specific pattern and to exert oncogenic or tumor suppressive functions in different types of cancer in humans (11-14). The downregulation of TUG1 has been detected in glioma and non-small-cell lung cancer (NSCLC), with TUG1 shown to induce apoptosis as a tumor suppressor $(15,16)$. By contrast, the overexpression of TUG1 has been reported in osteosarcoma (17), bladder cancer $(18,19)$, colorectal cancer (CRC) $(20)$, esophageal squamous cell carcinoma (ESCC) (21), gastric cancer (22) and hepatocellular cancer (HCC) (23). TUG1 was shown to function as an oncogene by promoting cell proliferation and was correlated with a poor prognosis (17-23).

Polycomb repressive complex 2 (PRC2) is a methyltransferase, which is composed of enhancer of zeste homolog 2 (EZH2), suppressor of zeste 12 (SUZ12) and embryonic ectoderm development, and is capable of catalyzing the di- and trimethylation of lysine residue 27 of histone 3 (H3K27me3), which regulates gene expression. Various lncRNAs, including TUG1, modulate specific genetic loci by recruiting and binding to $\mathrm{PRC} 2$ protein complexes, and PRC2-mediated epigenetic regulation is vital in tumorigenesis and development (24-27). The knockdown of TUG1 results in wide changes in gene expression, particularly the upregulation of cell-cycle genes, indicating that TUG1 is important in cell proliferation and apoptosis through effects on the cell cycle (22). However, the comprehensive mechanisms remain to be fully elucidated. 


\section{TUG1 in human cancer}

Colorectal cancer (CRC). It was previously reported that the expression of TUG1 was significantly enhanced in CRC tumor tissues, compared with that in paratumor tissues. Further analysis showed that the expression of TUG1 was negatively correlated with overall survival rates in patients (20). The stable knockdown of histone deacetylase 1 (HDAC1) induced the expression of TUG1, indicating that the expression of TUG1 is regulated by histone modification $(20,28)$. In vitro experiments have confirmed that the knockdown of TUG1 suppresses the colony formation, migration and invasion of CRC cells in vitro. In addition, an in vivo liver metastasis model revealed that the overexpression of TUG1 increased the number of metastatic tumor nodules in the liver, indicating that TUG1 promoted CRC metastasis (20). The molecular mechanism by which TUG1 promotes the invasion and metastasis of CRC has also been investigated. It was demonstrated that the overexpression of TUG1 reduced the expression of E-cadherin, and upregulated the expression levels of $\mathrm{N}$-cadherin, vimentin and fibronectin, whereas knockdown of the expression of TUG1 showed the opposite effects. This suggested that TUG1 may affect CRC metastasis and invasion through mediating epithelial-mesenchymal transition (EMT)-associated gene expression (20,29). However, the mechanisms by which HDAC1 affects TUG1 and regulates EMT require further investigation.

Bladder cancer. The expression of TUG1 was also found to be upregulated in bladder cancer tissues and cell lines. A higher expression of TUG1 was found to be associated with poorer tumor-necrosis-metastasis (TNM) staging and shorter overall survival rates $(18,19)$. Subsequent investigations revealed that TUG1 promoted bladder cancer cell invasion and radioresistance. The expression level of epithelial markers increased whereas those of mesenchymal markers decreased following the overexpression of TUG1, indicating that TUG1 was involved in bladder cancer through EMT $(19,29)$. TUG1 acted as a microRNA (miRNA) sponge, as miRNA (miR)-145 was able to bind to TUG1 and exhibit reciprocal regulatory effects. In addition, Zinc finger E-box binding homeobox 2 (ZEB2), a transcription factor regulating the EMT marker E-cadherin (30), has been identified as a direct target of miR-145 (31). The evidence above indicates a possible mechanism by which TUG1 is involved in EMT and the radioresistance in bladder cancer through the miR-145/ZEB2 axis.

Hepatocellular cancer (HCC). A previous study detected an upregulation in the expression of TUG1 in HCC tissues, which was confirmed to be associated with tumor size and Barcelona Clinic Liver Cancer stage (23).The transcription factor, stimulatory protein 1 (SP1) was later confirmed to directly bind to TUG1 promoter regions and positively regulate the expression of TUG1. In previous in vitro and xenograft model experiments, the functions of TUG1 in inhibiting cell proliferation and inducing cell apoptosis in HCC were demonstrated. Kruppel-like factor 2 (KLF2) was identified as a novel downstream gene of TUG1, which was found to be involved in HCC cell G0/G1 arrest, suppression of cell proliferation and the induction of apoptosis. TUG1 inhibited the transcription of KLF2 through binding to EZH2/SUZ12, the core subunits

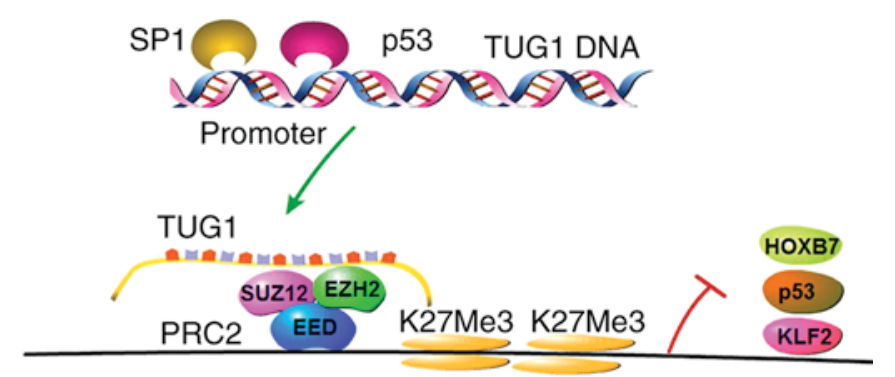

Tumor development and metastasis.

Figure 1. Schematic illustration of TUG1 binding to PRC2 in the regulation of tumor development and metastasis. TUG1, taurine-upregulated gene 1; PRC2, polycomb repressive complex 2; SP1, stimulatory protein 1; SUZ12, suppressor of zeste 12; EZH2, enhancer of zeste homolog 2; EED, embryonic ectoderm development; K27Me3, trimethylated histone $\mathrm{H} 3$ at lysine 27; HOXB7, homeobox B7; KLF2, Kruppel-like factor.

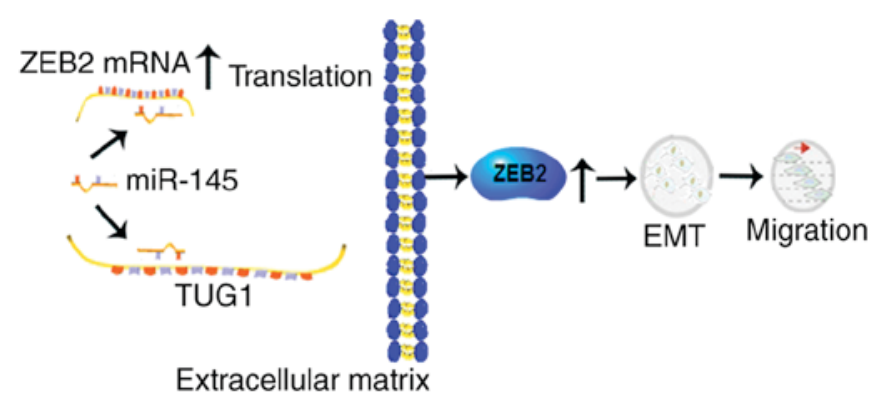

Figure 2. Schematic illustration of TUG1 altering EMT through miR-145 in the regulation of tumor development and metastasis. TUG1, taurine-upregulated gene 1; miR-145, microRNA-145; ZEB2, Zinc finger E-box binding homeobox 2; EMT, epithelial-mesenchymal transition.

of PRC2, to the KLF2 gene promoter locus in HCC cells, thus acting as an oncogenic factor (32).

Gastric cancer $(G C)$. A previous study observed that TUG1 was overexpressed in GC cells, and was positively correlated with invasion depth and TNM stage, but negatively correlated with overall survival rates (22). In vitro and in vivo experiments confirmed that TUG1 suppressed GC cell proliferation through its effects on cell cycle progression in a pattern of G0/G1 arrest. Subsequent investigation of the mechanism demonstrated that TUG1 specifically targeted EZH2 and epigenetically regulated the expression of cyclin-dependent kinase inhibitor (CKI) family members, including p15, p16, p21, p27 and p57 $(22,33,34)$. Analysis of the mechanism showed that TUG1 epigenetically repressed CKIs by binding to PRC2, and thereby regulated the cell cycle to promote GC cell proliferation.

ESCC. It was previously found that TUG1 was overexpressed in ESCC. Patients with a family history of esophageal cancer and upper segment ESCC were shown to express higher levels of TUG1. TUG1 also promoted the proliferation and migration of ESCC cell lines in vitro (21).

Osteosarcoma. TUG1 is upregulated in osteosarcoma tissues and cells (17). Experiments have demonstrated that 


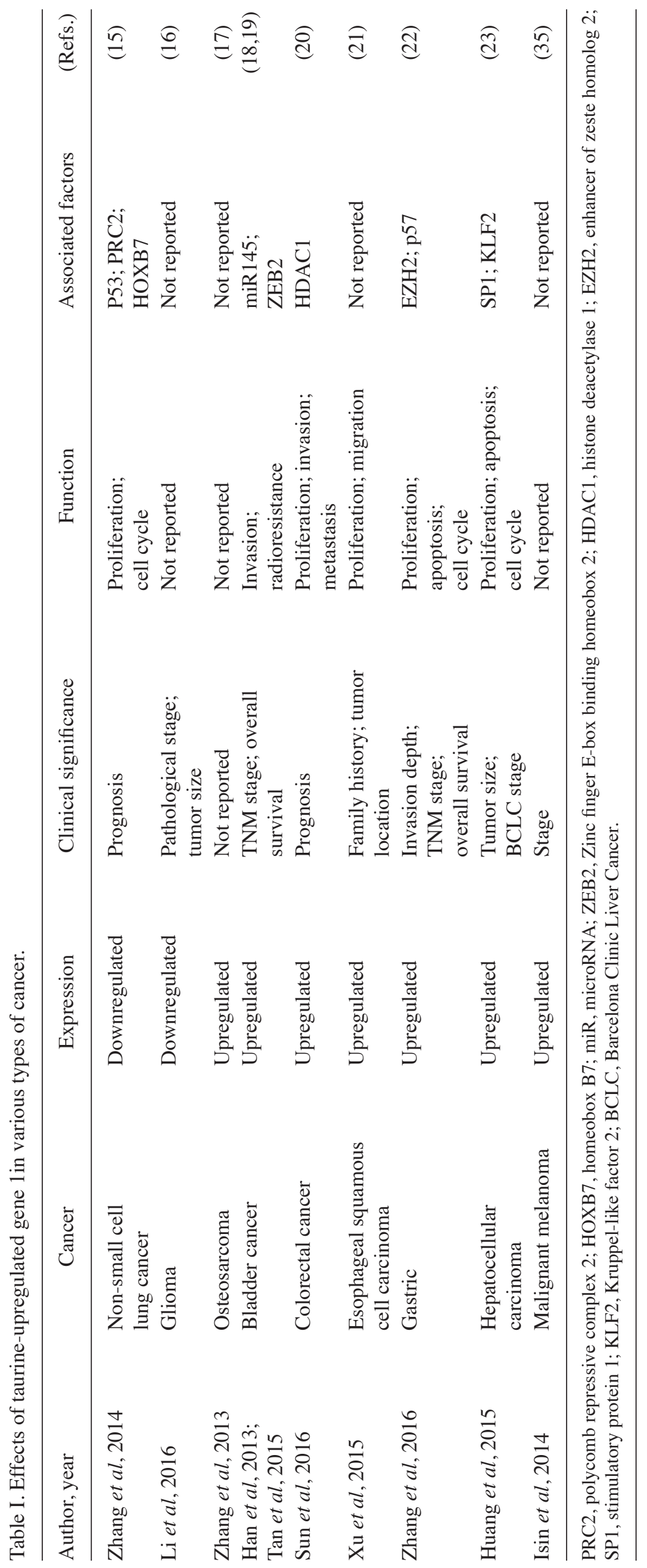


TUG1 acts as an oncogenic gene via increasing osteosarcoma cell proliferation and affecting apoptosis. However, the detailed mechanisms require further investigation.

Multiple myeloma (MM). It has been reported that, in the plasma of patients with MM, the expression of TUG1 was upregulated and showed marked association with clinical stages (35).

Glioma. Unlike the overexpression of TUG1 found in the types of cancer described above, TUG1 was downregulated in human glioma tissues and cells, and negatively associated with advanced pathological progression, serving as an indicator of poor prognosis (16). TUG1 exerts its antitumor function in glioma through promoting cell apoptosis via intrinsic pathways mediated by caspase- 3 together with caspase- 9 , and simultaneously suppressing anti-apoptotic pathways mediated by B-cell lymphoma 2 (36-39).

Non-small-cell lung cancer (NSCLC). The expression of TUG1 was also shown to be downregulated in human NSCLC tissues and correlated with poor prognosis of patients (15). TUG1 has been shown to modulate NSCLC cell proliferation in vitro and in vivo through alterations in cell cycle progression. Further analysis demonstrated that p53 was able to directly bind to the promoter region of TUG1 and regulate the expression of TUG1. In addition, investigations have suggested that homeobox B7 (HOXB7), a known oncogene, is a downstream gene of TUG1, and it was suggested that TUG1 targets PRC2 to regulate HOXB7 at the transcriptional level $(40,41)$. The role of HOXB7 has also been investigated, which showed that HOXB7 promoted NSCLC cell proliferation via activating the AKT and mitogen-activated protein kinase pathways $(15,38,39,42,43)$. Therefore, the p53/TUG1/PRC2/HOXB7 axis was found to be vital in the tumorigenesis and progression of NSCLC, which may be a target for future therapy.

\section{Conclusions and perspectives}

In previous years, widespread investigations have been performed on the biological roles and clinical significance of lncRNAs. The deregulation of lncRNAs is capable of affecting tumor development, functioning as tumor inducers or suppressors. One of the most comprehensively investigated lncRNAs is the hox transcript antisense intergenic RNA (HOTAIR). HOTAIR has been identified as an oncogenic gene, recruiting PRC2 and interacting with lysine-specific demethylase1 (LSD1), regulating the expression of its downstream targets $(8,11,42)$.

This review discusses TUG1 and its association with cancer in humans. TUG1 is expressed in a tissue-specific pattern, showing oncogenic or tumor inhibiting capacities in different types of cancer in humans (Table I). The downregulation of TUG1 is observed in glioma and NSCLC, showing that TUG1 serves as a tumor suppressor. By contrast, the overexpression of TUG1 has been reported in ESCC, CRC, HCC, bladder cancer and osteosarcoma, indicating that TUG1 functions as an oncogene. The mechanisms underlying the biological functions of TUG1 are shown in Figs. 1 and 2. These findings indicate that the expression of TUG1 can be enhanced or weakened by upstream or downstream interference to slow down or alter tumor progression. The level of TUG1 in tumor tissues correlates with tumor stage and prognosis, which may be utilized as a diagnostic and prognostic biomarker clinically. In a previous study, TUG1 was moderately elevated in secreted exosomes (44), and this may enable the isolation of exosomes from blood plasma or serum to assist in monitoring the state of disease dynamically (45). However, further investigations are required in order to fully elucidate the mechanisms underlying TUG1 and cancer development. TUG1 may offer potential as a novel diagnostic biomarker and therapeutic approach for clinical utilization.

\section{Acknowledgements}

This review was supported by grants from National Natural Science Foundation of China (grant no. 81272532).

\section{References}

1. Derrien T, Johnson R, Bussotti G, Tanzer A, Djebali S, Tilgner H, Guernec G, Martin D, Merkel A, Knowles DG, et al: The GENCODE v7 catalog of human long noncoding RNAs: Analysis of their gene structure, evolution, and expression. Genome Res 22: 1775-1789, 2012.

2. Mercer TR, Dinger ME and Mattick JS: Long non-coding RNAs: Insights into functions. Nat Rev Genet 10: 155-159, 2009.

3. An integrated encyclopedia of DNA elements in the human genome. Nature 489: 57-74, 2012.

4. Amaral PP and Mattick JS: Noncoding RNA in development. Mamm Genome 19: 454-492, 2008.

5. Blackshaw S, Harpavat S, Trimarchi J, Cai L, Huang H, Kuo WP, Weber G, Lee K, Fraioli RE, Cho SH, et al: Genomic analysis of mouse retinal development. PLoS Biol 2: e247, 2004.

6. Dinger ME, Amaral PP, Mercer TR, Pang KC, Bruce SJ, Gardiner BB, Askarian-Amiri ME, Ru K, Soldà G, Simons $\mathrm{C}$, et al: Long noncoding RNAs in mouse embryonic stem cell pluripotency and differentiation. Genome Res 18: 1433-1445, 2008.

7. Cesana M, Cacchiarelli D, Legnini I, Santini T, Sthandier O, Chinappi M, Tramontano A and Bozzoni I: A long noncoding RNA controls muscle differentiation by functioning as a competing endogenous RNA. Cell 147: 358-369, 2011.

8. Rinn JL, Kertesz M, Wang JK, Squazzo SL, Xu X, Brugmann SA, Goodnough LH, Helms JA, Farnham PJ, Segal E and Chang HY: Functional demarcation of active and silent chromatin domains in human HOX loci by noncoding RNAs. Cell 129: 1311-1323, 2007.

9. Ginger MR, Shore AN, Contreras A, Rijnkels M, Miller J, Gonzalez-Rimbau MF and Rosen JM: A noncoding RNA is a potential marker of cell fate during mammary gland development. Proc Natl Acad Sci USA 103: 5781-5786, 2006.

10. Young TL, Matsuda T and Cepko CL: The noncoding RNA taurine upregulated gene 1 is required for differentiation of the murine retina. Curr Biol 15: 501-512, 2005.

11. Gupta RA, Shah N, Wang KC, Kim J, Horlings HM, Wong DJ, Tsai MC, Hung T, Argani P, Rinn JL, et al: Long non-coding RNA HOTAIR reprograms chromatin state to promote cancer metastasis. Nature 464: 1071-1076, 2010.

12. Khaitan D, Dinger ME, Mazar J, Crawford J, Smith MA, Mattick JS and Perera RJ: The melanoma-upregulated long noncoding RNA SPRY4-IT1 modulates apoptosis and invasion. Cancer Res 71: 3852-3862, 2011.

13. Yuan SX, Yang F, Yang Y, Tao QF, Zhang J, Huang G, Yang Y, Wang RY, Yang S, Huo XS, et al: Long noncoding RNA associated with microvascular invasion in hepatocellular carcinoma promotes angiogenesis and serves as a predictor for hepatocellular carcinoma patients' poor recurrence-free survival after hepatectomy. Hepatology 56: 2231-2241, 2012.

14. Ji P, Diederichs S, Wang W, Böing S, Metzger R, Schneider PM, Tidow N, Brandt B, Buerger H, Bulk E, et al: MALAT-1, a novel noncoding RNA and thymosin beta4 predict metastasis and survival in early-stage non-small cell lung cancer. Oncogene 22: 8031-8041, 2003 
15. Zhang EB, Yin DD, Sun M, Kong R, Liu XH, You LH, Han L, Xia R, Wang KM, Yang JS, et al: P53-regulated long non-coding RNA TUG1 affects cell proliferation in human non-small cell lung cancer, partly through epigenetically regulating HOXB7 expression. Cell Death Dis 5: e1243, 2014.

16. Li J, Zhang M, An G and Ma Q: LncRNA TUG1 acts as a tumor suppressor in human glioma by promoting cell apoptosis. Exp Biol Med (Maywood) 241: 644-649, 2016.

17. Zhang Q, Geng PL, Yin P, Wang XL, Jia JP and Yao J: Down-regulation of long non-coding RNA TUG1 inhibits osteosarcoma cell proliferation and promotes apoptosis. Asian Pac J Cancer Prev 14: 2311-2315, 2013.

18. Han Y, Liu Y, Gui Y and Cai Z: Long intergenic non-coding RNA TUG1 is overexpressed in urothelial carcinoma of the bladder. J Surg Oncol 107: 555-559. 2013.

19. Tan J, Qiu K, Li M and Liang Y: Double-negative feedback loop between long non-coding RNA TUG1 and miR-145 promotes epithelial to mesenchymal transition and radioresistance in human bladder cancer cells. FEBS Lett 589: 3175-3181, 2015.

20. Sun J, Ding C, Yang Z, Liu T, Zhang X, Zhao C and Wang J: The long non-coding RNA TUG1 indicates a poor prognosis for colorectal cancer and promotes metastasis by affecting epithelial-mesenchymal transition. J Transl Med 14: 42, 2016.

21. Xu Y, Wang J, Qiu M, Xu L, Li M, Jiang F, Yin R and Xu L: Upregulation of the long noncoding RNA TUG1 promotes proliferation and migration of esophageal squamous cell carcinoma. Tumour Biol 36: 1643-1651, 2015.

22. Zhang E, He X, Yin D, Han L, Qiu M, Xu T, Xia R, Xu L, Yin R and De W: Increased expression of long noncoding RNA TUG1 predicts a poor prognosis of gastric cancer and regulates cell proliferation by epigenetically silencing of p57. Cell Death Dis 7: e2109, 2016.

23. Huang MD, Chen WM, Qi FZ, Sun M, Xu TP, Ma P and Shu YQ: Long non-coding RNA TUG1 is up-regulated in hepatocellular carcinoma and promotes cell growth and apoptosis by epigenetically silencing of KLF2. Mol Cancer 14: 165, 2015.

24. Paul TA, Bies J, Small D and Wolff L: Signatures of polycomb repression and reduced $\mathrm{H} 3 \mathrm{~K} 4$ trimethylation are associated with p15INK4b DNA methylation in AML. Blood 115: 3098-3108, 2010.

25. Aoki R, Chiba T, Miyagi S, Negishi M, Konuma T, Taniguchi H, Ogawa $\mathrm{M}$, Yokosuka $\mathrm{O}$ and Iwama $\mathrm{A}$ : The polycomb group gene product Ezh2 regulates proliferation and differentiation of murine hepatic stem/progenitor cells. J Hepatol 52: 854-863, 2010.

26. Chen H, Gu X, Su IH, Bottino R, Contreras JL, Tarakhovsky A and Kim SK: Polycomb protein Ezh2 regulates pancreatic beta-cell Ink4a/Arf expression and regeneration in diabetes mellitus. Genes Dev 23: 975-985, 2009.

27. Fan T, Jiang S, Chung N, Alikhan A, Ni C, Lee CC and Hornyak TJ: EZH2-dependent suppression of a cellular senescence phenotype in melanoma cells by inhibition of $\mathrm{p} 21 / \mathrm{CDKN} 1 \mathrm{~A}$ expression. Mol Cancer Res 9: 418-429, 2011.

28. Zilio N, Codlin S, Vashisht AA, Bitton DA, Head SR, Wohlschlegel JA, Bähler J and Boddy MN: A novel histone deacetylase complex in the control of transcription and genome stability. Mol Cell Biol 34: 3500-3514, 2014.

29. Thiery JP, Acloque H, Huang RY and Nieto MA: Epithelial-mesenchymal transitions in development and disease. Cell 139: 871-890, 2009.
30. Vandewalle C, Comijn J, De Craene B, Vermassen P, Bruyneel E, Andersen H, Tulchinsky E, Van Roy F and Berx G: SIP1/ZEB2 induces EMT by repressing genes of different epithelial cell-cell junctions. Nucleic Acids Res 33: 6566-6578, 2005.

31. Ambros V: The functions of animal microRNAs. Nature 431: 350-355, 2004

32. Fernandez-Zapico ME, Lomberk GA, Tsuji S, DeMars CJ, Bardsley MR, Lin YH, Almada LL, Han JJ, Mukhopadhyay D, Ordog T, et al: A functional family-wide screening of SP/KLF proteins identifies a subset of suppressors of KRAS-mediated cell growth. Biochem J 435: 529-537, 2011.

33. Kotake Y, Nakagawa T, Kitagawa K, Suzuki S, Liu N, Kitagawa M and Xiong Y: Long non-coding RNA ANRIL is required for the PRC2 recruitment to and silencing of p15(INK4B) tumor suppressor gene. Oncogene 30: 1956-1962, 2011

34. Prensner JR, Iyer MK, Balbin OA, Dhanasekaran SM, Cao Q, Brenner JC, Laxman B, Asangani IA, Grasso CS, Kominsky HD, et al: Transcriptome sequencing across a prostate cancer cohort identifies PCAT-1, an unannotated lincRNA implicated in disease progression. Nat Biotechnol 29: 742-749, 2011.

35. Isin M, Ozgur E, Cetin G, Erten N, Aktan M, Gezer U and Dalay $\mathrm{N}$ : Investigation of circulating lncRNAs in B-cell neoplasms. Clin Chim Acta 431: 255-259, 2014.

36. Ghavami S, Hashemi M, Ande SR, Yeganeh B, Xiao W, Eshraghi M, Bus CJ, Kadkhoda K, Wiechec E, Halayko AJ and Los M: Apoptosis and cancer: Mutations within caspase genes. J Med Genet 46: 497-510, 2009.

37. Brentnall M, Rodriguez-Menocal L, De Guevara RL, Cepero E and Boise LH: Caspase-9, caspase-3 and caspase-7 have distinct roles during intrinsic apoptosis. BMC Cell Biol 14: 32, 2013.

38. Liao WT, Jiang D, Yuan J, Cui YM, Shi XW, Chen CM, Bian XW, Deng YJ and Ding YQ: HOXB7 as a prognostic factor and mediator of colorectal cancer progression. Clin Cancer Res 17: 3569-3578, 2011.

39. Jin K, Kong X, Shah T, Penet MF, Wildes F, Sgroi DC, Ma XJ, Huang Y, Kallioniemi A, Landberg G, et al: The HOXB7 protein renders breast cancer cells resistant to tamoxifen through activation of the EGFR pathway. Proc Natl AcadSci USA 109: 2736-2741, 2012

40. Storti P, Donofrio G, Colla S, Airoldi I, Bolzoni M, Agnelli L, Abeltino M, Todoerti K, Lazzaretti M, Mancini C, et al: HOXB7 expression by myeloma cells regulates their pro-angiogenic properties in multiple myeloma patients. Leukemia 25: 527-537, 2011.

41. Yuan W, Zhang X, Xu Y, Li S, Hu Y and Wu S: Role of HOXB7 in regulation of progression and metastasis of human lung adenocarcinoma. Mol Carcinog 53: 49-57, 2014.

42. di Pietro M, Lao-Sirieix P, Boyle S, Cassidy A, Castillo D, Saadi A, Eskeland R and Fitzgerald RC: Evidence for a functional role of epigenetically regulated midcluster HOXB genes in the development of Barrett esophagus. Proc Natl Acad Sci USA 109: 9077-9082, 2012.

43. Wu X, Chen H, Parker B, Rubin E, Zhu T, Lee JS, Argani P and Sukumar S: HOXB7, a homeodomain protein, is overexpressed in breast cancer and confers epithelial-mesenchymal transition. Cancer Res 66: 9527-9534, 2006

44. Gezer U, Özgür E, Cetinkaya M, Isin M and Dalay N: Long non-coding RNAs with low expression levels in cells are enriched in secreted exosomes. Cell Biol Int 38: 1076-1079, 2014.

45. Bang C and Thum T: Exosomes: New players in cell-cell communication. Int J Biochem Cell Biol 44: 2060-2064, 2012. 